\title{
Efficient On-Chip Phonon-Photon Translation
}

\author{
Amir H. Safavi-Naeini, Thiago P. Mayer Alegre and Oskar J. Painter \\ Department of Applied Physics, California Institute of Technology, Pasadena, CA 91125, USA. \\ safavi@caltech.edu
}

\begin{abstract}
We propose, analyze, design, and take the first experimental steps towards the demonstration of an on-chip device capable of converting photons to phonons, and vice versa, in a nearly quantumlimited setting.

(c) 2010 Optical Society of America

OCIS codes: (130.0130) Integrated optics, (290.5830) Scattering, Brillouin
\end{abstract}

With new nanofabrication technology, and in particular, the advent of Optomechanical Crystals (OMC) [1, 2], the simultaneous localization and strong interaction of photons and phonons in a diffraction-limited setting enters the realm of possibility. Photonic and phononic information processing techniques have long been developed separately. While photonic information transmission networks have formed the back-bone of the internet, phononic information processing circuits in the form of Surface Acoustic Wave (SAW) and Bulk Acoustic Wave (BAW) devices have become ubiquitous in the world of wireless. In general, the advantages of photons, i.e. their lack of interaction with the environment (leading to low-loss fibers), their high velocities (long distance communication) and their large available bandwidth due to their intrinsically high energy, place limits on our ability to process them. For example, sharp (MHz-scale) optical add and drop filters are achievable with certain techniques, but are generally difficult to make. Optical delay lines are also hard to manufacture on an integrated platform and have long been subject to investigation. Phonons on the other hand are slow, have lower energy, and interact more strongly with certain parts of their environment. This makes them bad carriers of information, but at the same time, complimentary in many respects to photons. We propose a method [3] to convert photons to phonons efficiently in a quantum-limited setting, using a device called a Phonon-Photon Translator (PPT).

(a)
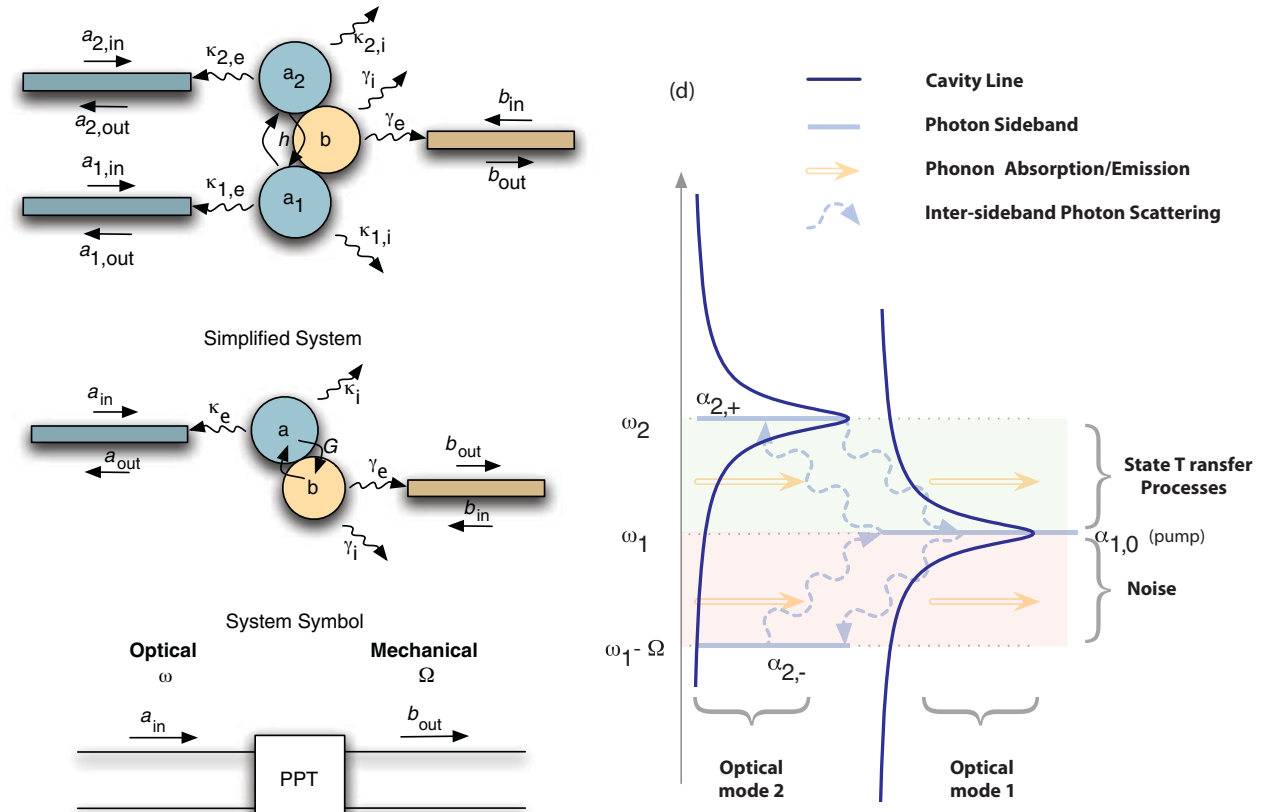

Fig. 1. The full system (a) is presented along with the simplified approximate model (b), (c). In (d) Relevant sidebands and processes are shown for the double cavity system. The pump is on sideband $\alpha_{1,0}$, at the same frequency as the first cavity resonance $\omega_{1}$. We see that there are three sidebands, $\alpha_{2,+}, \alpha_{1,0}$ and $\alpha_{2,-}$. The inter-sideband photon scattering can give rise to phonon emission and absorption as is represented by the three-level system. The state transfer occurs through scattering between sidebands $\alpha_{2,+}$ and $\alpha_{1,0}$. All inter-sideband scattering between $\alpha_{1,0}$ and $\alpha_{2,-}$ can be thought of as phonon noise. Note that there are no sidebands at $\omega_{2}$ and $\omega_{1}-\Omega$ for cavity 1 , and no sideband at $\omega_{1}$ for cavity 2 ; they have been eliminated through careful use of selection rules. 
The canonical optomechanical interaction is a non-linear coupling between two harmonic oscillators. When one sideband (the pump) is at a much higher intensity than all other sidebands, a situation which can be achieved by properly setting the various system parameters, an effectively linear coupling between the mechanical oscillator and the "signal" sideband can be achieved. This is represented by the green shaded area in Figure 1(d). Many of the unique features of cavity optomechanics arise from the non-uniform colouring of the vacuum by a photonic density of states of the cavity. For example, cooling of the mechanical mode arises from the non-uniform inter-sideband scattering rates into the stokes and anti-stokes sidebands of an optical pump. By using two optical modes as opposed to one, we engineer further the interesting aspects of our cavity optomechanical system. For example, we use two optical cavity modes, leading to an order of magnitude reduction in the required pump input power. Also, by eliminating first order self-coupling sidebands through careful engineering of the optical and mechanical mode profiles, we reduce the noise which is imparted on the system. This reduction, though not particularly relevant for technological applications of the PPT, becomes important in the regime where the system is cooled to $\mathrm{mK}$ temperatures.

(a)

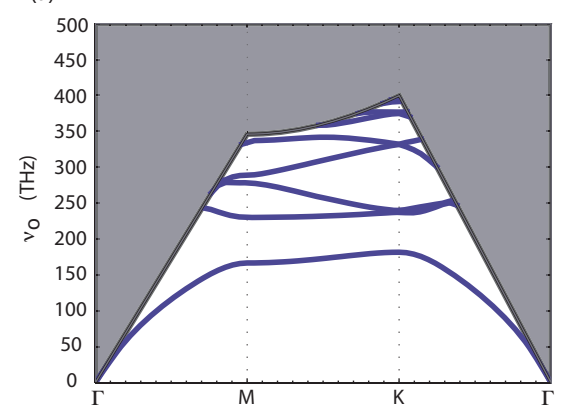

(b)

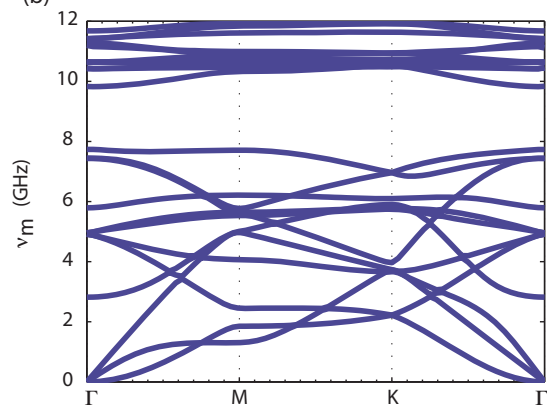

(c)

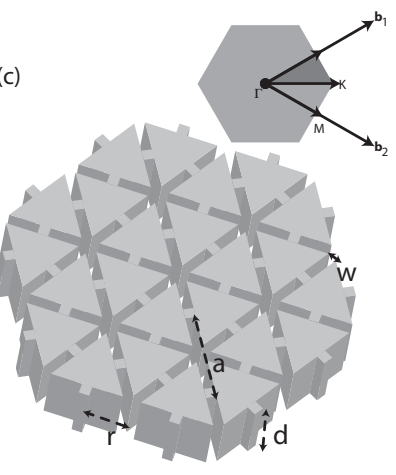

Fig. 2. The snowflake crystal (c) used as the substrate on which the PPT is to be implemented is demonstrated along with (a) optical and (b) mechanical band diagrams for parameters $(\mathrm{d}, \mathrm{r}, \mathrm{w}, \mathrm{a})=(220,200,75,500) \mathrm{nm}$.

The implementation of the PPT can be achieved on a quasi-2D photonic-phononic crystal slab supporting guided and confined phononic and photonic modes. We demonstrate how large simultaneous phononic-photonic bandgap crystals can be designed [4], and use one of these crystals, shown in Figure 2 to design an optomechanical cavity with a radiation-limited $Q$ exceeding $5 \times 10^{7}$, and an optomechanical coupling $\frac{g}{2 \pi}>250 \mathrm{kHz}$. Up-to-date experimental results will be provided.

Finally, we investigate applications of the PPT to problems in classical integrated optics and quantum information processing. On the quantum front, we show that a PPT may be used to implement a typical quantum state transfer process [5] between optical and superconducting qubits $[6,7]$ with a realistic fidelity of around 0.80 .

\section{References}

1. M. Eichenfield, J. Chan, R. M. Camacho, K. J. Vahala, and O. Painter, “Optomechanical crystals," Nature 462, 78-82 (2009). http://dx.doi.org/10.1038/nature08524

2. M. Eichenfield, J. Chan, A. H. Safavi-Naeini, K. J. Vahala, and O. Painter, "Modeling dispersive coupling and losses of localized optical and mechanical modes in optomechanical crystals," Opt. Express 17, 20 078-20 098 (2009). http://www.opticsexpress.org/abstract.cfm?URI=oe-17-22-20078

3. A. H. Safavi-Naeini, K. J. Vahala, and O. J. Painter, "Proposal for an On-Chip Phonon Photon Translator and Applications," (2009).

4. A. H. Safavi-Naeini and O. J. Painter, "Design of Ultrahigh- $Q$ Optomechanical Cavities and Waveguides on a Simultaneous Bandgap Phononic-Photonic Crystal Slab," (2009).

5. J. I. Cirac, P. Zoller, H. J. Kimble, and H. Mabuchi, "Quantum State Transfer and Entanglement Distribution among Distant Nodes in a Quantum Network," Phys. Rev. Lett. 78, 3221- (1997). http://link.aps.org/abstract/PRL/v78/p3221

6. A. N. Cleland and M. R. Geller, "Superconducting qubit storage and entanglement with nano-mechanical resonators," Phys. Rev. Lett. 93, $070501-(2004)$. http://dx.doi.org/10.1103/PhysRevLett.93.070501

7. M. R. Geller and A. N. Cleland, "Superconducting qubits coupled to nanoelectromechanical resonators: An architecture for solid-state quantum-information processing," Phys. Rev. A 71, 032 311- (2005).

http://link.aps.org/abstract/PRA/v71/e032311 